\title{
Association between serum uric acid and ischemic stroke in patients with nonvalvular paroxysmal atrial fibrillation
}

\author{
Nermin Bayar ${ }^{\mathrm{a}, *}$, Selcuk Küçükseymen ${ }^{\mathrm{a}}$, Ramazan Güven ${ }^{\mathrm{b}}$, Zehra Erkal ${ }^{\mathrm{a}}$, Erkan Köklü ${ }^{\mathrm{a}}$, İsa Öner Yüksel ${ }^{\mathrm{a}}$, \\ Göksel Çağırcl ${ }^{\text {a }}$, Şakir Arslan ${ }^{\text {a }}$
}

a Antalya Education and Research Hospital, Cardiology Department, Antalya, Turkey

b Bitlis State Hospital, Emergency Medicine Department, Antalya, Turkey

\section{A R T I C L E I N F O}

\section{Article history:}

Received 2 August 2016

Received in revised form 26 September 2016

Accepted 27 September 2016

Available online 28 September 2016

\section{Keywords:}

Stroke

Atrial fibrillation

Serum uric acid

\begin{abstract}
A B S T R A C T
Objective: In this study, we aimed to investigate the value of serum uric acid levels in predicting stroke and transient ischemic attacks among patients with paroxysmal AF.

Methods: We enrolled patients with paroxysmal AF. Clinical and demographic features of the patients were recorded, and $\mathrm{CHA}_{2} \mathrm{DS}_{2}$ VASC scores were calculated. Uric acid levels were also recorded from biochemistry results. Patient groups with and without history of stroke and transient ischemic attack were compared for their serum uric acid levels.

Results: A total of 180 patients were enrolled in this study, of whom 62 had a history of ischemic stroke/transient ischemic attack (symptomatic group). $\mathrm{CHA}_{2} \mathrm{DS}_{2}$ VASC score in the symptomatic group was $4.7 \pm 1.2$ while it was $2.6 \pm 1.4$ in the asymptomatic group $(<0.001)$. It was also shown that uric acid levels were higher in the symptomatic group when compared to the asymptomatic group ( $7.64 \pm 1.12 \mathrm{mg} / \mathrm{dl} \mathrm{vs} 5.06 \pm 1.16 \mathrm{mg} / \mathrm{dl}$, $p<0.001$, respectively). Multivariate analysis revealed that a serum uric acid level higher than $6.35 \mathrm{mg} / \mathrm{dl}$ was independently associated with having a history of stroke/transient ischemic attack.

Conclusion: Assessment of uric acid levels in addition to $\mathrm{CHA}_{2} \mathrm{DS}_{2}$ VASC score might be useful in patients with paroxysmal AF to select patients with higher risk of stroke/transient ischemic attack.

(C) 2016 The Society of Cardiovascular Academy. Production and hosting by Elsevier B.V. All rights reserved. This is

an open access article under the CC BY-NC-ND license (http://creativecommons.org/licenses/by-nc-nd/4.0/).
\end{abstract}

\section{Introduction}

Atrial fibrillation (AF) is a major reason for cardioembolic stroke. Since stroke has a poor prognosis among patients with AF, it is important to recognize patients with high stroke risk. A common practice to assess stroke risk in AF patients is $\mathrm{CHA}_{2} \mathrm{DS}_{2}$ VASC score. ${ }^{1}$ However, recent studies showed that patients with lower $\mathrm{CHA}_{2} \mathrm{DS}_{2}$ VASC scores might also have high risk for thromboembolism. ${ }^{2,3}$ Therefore, determination of additional risk factors is necessary to recognize patients with true low risk.

Prospective studies have shown that uric acid is associated with inflammation and oxidative stress, both of which also operate in the pathogenesis of $\mathrm{AF}^{4-6}$ It was reported that the risk for development of stroke is high among AF patients with high serum uric acid (SUA) levels and hyperuricemia was also associated with higher rates of early mortality among stroke patients. ${ }^{2,7}$ In this study, we aimed to investigate

\footnotetext{
* Corresponding author at: Antalya Education and Research Hospital, Cardiology Department, Muratpasa, 07100 Antalya, Turkey.

E-mail address: dr.nermin@mynet.com (N. Bayar).

Peer review under responsibility of The Society of Cardiovascular Academy.
}

the usefulness of SUA assessment in paroxysmal AF patients in predicting the risk for stroke and transient ischemic attack (TIA).

\section{Methods}

We enrolled non-rheumatismal paroxysmal AF patients who applied to and were diagnosed in our clinic between 2012 and 2015, among whom none had anticoagulant treatment. Baseline demographic features were registered. PAF diagnosis was assigned based on 12-lead ECG or Holter electrocardiography recordings. AF attacks terminating spontaneously in a period shorter than 7 days were considered to be PAF. Patients with heart failure, moderate to severe valvular disease and thyroid dysfunction, which was not euthyroid, were excluded from this study. Patients with inflammatory or metabolic diseases that disturb uric acid metabolism, electrolyte imbalance, previous myocardial infarction, collagen tissue disease, cardiomyopathy and congenital heart diseases were also excluded from this study. Patients' $\mathrm{CHA}_{2} \mathrm{DS}_{2}$ VASc scores were calculated. ${ }^{8}$ Stroke was defined as brain, spinal cord or retinal cell death attributable to ischemia based on neuropathological, neuroimaging and/or clinical evidence of permanent injury. Transient ischemic attacks are episodes of temporary and focal dysfunction of vascular origin, which are variable in duration, commonly lasting from 
2 to $15 \mathrm{~min}$, but occasionally lasting as long as a day (24 h). ${ }^{9}$ Patients who had a history of stroke or TIA were defined as the symptomatic group while those who did not have such history were defined as the asymptomatic group. Participants gave their written informed consent. Approval was obtained from the local ethics board for this study.

Electrocardiography (ECG) with 12 derivations (Nihon Kohden, Tokyo, Japan) was performed for all patients. All patients were subjected to 2-dimensional, M-mode, pulsed and color Doppler echocardiographic examinations (Philips EPIQ 7 Cardiac Ultrasound) by the same cardiologist who was incognisant concerning the history of stroke/TIA of patients. During echocardiography, a single lead (D2) electrocardiogram was recorded simultaneously. Data were recorded from the average of three cardiac cycles. M-mode and Doppler measurements were performed by the same cardiologist according to the American Society of Echocardiography guidelines. ${ }^{10}$ All measurements were recorded over an average of at least 3 cycles.

$\mathrm{CHA}_{2} \mathrm{DS}_{2}$ VASC scores were calculated to assess the risk of thromboembolism. ${ }^{1}$ Venous blood was withdrawn in the morning after an overnight fast. Lipids, creatinine and other biochemical blood measurements were determined by standard laboratory procedures. SUA levels were recorded from routine biochemical tests. In addition, neutrophil: lymphocyte and platelet:lymphocyte ratios were calculated as inflammatory markers.

\section{Statistical analysis}

Data were analyzed with the SPSS software version 15.0 for Windows (SPSS Inc., Chicago, IL, USA). Categorical variables were presented as frequency and percentage. The $\chi^{2}$ test and Fisher's exact test were used to compare categorical variables. The KolmogorovSmirnov test was used to assess the distribution of continuous variables. Student's $t$-test was used for variables with normal distribution and the values were presented as mean $\pm \mathrm{SD}$. Continuous variables without normal distribution were analyzed using Mann-Whitney $U$ test and obtained values were presented as median (50th) values and interquantile ranges (25th and 75th). The odds ratios (OR) and 95\% confidence intervals $(\mathrm{CI})$ were calculated. A two-tailed $p$-value of $<0.05$ was considered statistically significant.

\section{Results}

Sixty-two out of 180 patients enrolled in this study had a history of ischemic stroke/transient ischemic attack (symptomatic group). Mean age was $63.3 \pm 3.0$ years in the symptomatic group while it was $61.4 \pm 3.2$ years in the asymptomatic group $(p=0.128)$ (Table 1$)$.

$\mathrm{CHA}_{2} \mathrm{DS}_{2}$ VASC score in the symptomatic group was $4.7 \pm 1.2$ while it was $2.6 \pm 1.4$ in the asymptomatic group $(p<0.001)$. However, when 2 points were subtracted from $\mathrm{CHA}_{2} \mathrm{DS}_{2}$ VASC scores in the symptomatic

Table 1

Clinical characteristics of the study population.

\begin{tabular}{llll}
\hline Variables & $\begin{array}{l}\text { Stroke/TIA }(+) \\
(n=62)\end{array}$ & $\begin{array}{l}\text { Stroke/TIA }(-) \\
(n=118)\end{array}$ & P value \\
\hline Age (years) & $63.3 \pm 3.0$ & $61.4 \pm 3.2$ & 0.128 \\
$>$ 65 years & $19(62)$ & $24(40 \%)$ & 0.039 \\
Male, $n(\%)$ & $38(61)$ & $50(42)$ & 0.046 \\
Body mass index $\left(\mathrm{kg} / \mathrm{m}^{2}\right)$ & $23.5 \pm 2.1$ & $23.1 \pm 2.8$ & 0.381 \\
Hypertension, $n(\%)$ & $52(84)$ & $100(85)$ & 1.000 \\
Diabetes mellitus, $n(\%)$ & $20(32)$ & $28(24)$ & 0.455 \\
Coronary artery disease, $n(\%)$ & $38(61)$ & $40(34)$ & 0.015 \\
Dyslipidemia, $n(\%)$ & $30(48)$ & $42(36)$ & 0.265 \\
Smoking, $n(\%)$ & $18(29)$ & $28(24)$ & 0.617 \\
CHA2DS2VASc score & $4.7 \pm 1.2$ & $2.6 \pm 1.4$ & $<0.001$ \\
Serum uric acid, mg/dl & $7.64 \pm 1.12$ & $5.06 \pm 1.16$ & $<0.001$ \\
\hline
\end{tabular}

Data are expressed as mean \pm standard deviation for normally distributed data and percentage for categorical variables.
Table 2

Conventionally transthoracic echocardiographic parameters of the study population.

\begin{tabular}{llll}
\hline Variables & $\begin{array}{l}\text { Stroke/TIA }(+) \\
(n=62)\end{array}$ & $\begin{array}{l}\text { Stroke/TIA }(-) \\
(n=118)\end{array}$ & P value \\
\hline LVEF, \% & $60(58-64)$ & $62(60-65)$ & 0.321 \\
LVEDD, mm & $49.3 \pm 7.0$ & $46.1 \pm 4.4$ & 0.009 \\
LVESD, mm & $32.9 \pm 8.9$ & $29.6 \pm 6.4$ & 0.050 \\
LAD, mm & $46 \pm 6.1$ & $41 \pm 7.0$ & 0.002 \\
LVH presence, $n(\%)$ & $34(55)$ & $70(58)$ & 1.000 \\
Mitral E/A & $0.84(0.6-1.5)$ & $1.4(0.7-1.7)$ & 0.019 \\
Mitral EDT, ms & $225 \pm 70$ & $213 \pm 59$ & 0.444 \\
IVRT, ms & $112 \pm 16$ & $108 \pm 22$ & 0.417 \\
Aortic root, mm & $32.7 \pm 3.9$ & $31.4 \pm 4.6$ & 0.225 \\
\hline
\end{tabular}

The table shows a normal distribution mean \pm SD, but does not show a normal distribution median (25th vs 75th percentile). EDT, E wave deceleration time; IVRT, isovolumic relaxation time; LAD, left atrium diameter; parasternal long axis LVEF, left ventricular ejection fraction; LVEDD, left ventricular end diastolic diameter; LVESD, left ventricular end systolic diameter; LVH, left ventricular hypertrophy.

group due to their stroke history, there was no significant difference between the two groups for $\mathrm{CHA}_{2} \mathrm{DS}_{2}$ VASC score $(2.7 \pm 1.2$ vs $2.6 \pm 1.4$, $p=0.07$, respectively).

There were no differences between two groups for conventional echocardiographic parameters, including left ventricular ejection fraction and left ventricular hypertrophy. However, the symptomatic group had higher left atrial diameter, left ventricular end-diastolic and end-systolic diameters (Table 2).

SUA level was higher in the symptomatic group as compared to asymptomatic group $(7.64 \pm 1.12 \mathrm{mg} / \mathrm{dl}$ vs $5.06 \pm 1.16 \mathrm{mg} / \mathrm{dl}$, $p<0.001$, respectively). Multivariate logistic regression analysis revealed that SUA levels higher than or equal to $6.35 \mathrm{mg} / \mathrm{dl}$ were independently associated with the history of stroke/transient ischemic attack $(\mathrm{OR}=81.623 ;$ 95\% CI: 17.33-384.26, $p<0.001)$ (Table 3).

\section{Discussion}

In this study, we showed that SUA levels equal to or higher than $6.35 \mathrm{mg} / \mathrm{dl}$ were independently associated with the history of stroke/ transient ischemic attack among patients with paroxysmal AF. This result indicates that SUA measurement might have an additional value in recognizing paroxysmal AF patients with higher stroke risk.

AF is a major cause of ischemic strokes. AF-related strokes have a dismal prognosis and more than $50 \%$ of survivors have severe deficits with a recurrence rate as high as $12 \%{ }^{11}$ Therefore, it is important to recognize high-stroke risk paroxysmal AF patients earlier. Actual guidelines recommend $\mathrm{CHA}_{2} \mathrm{DS}_{2}$ VASC score to assess the risk of thromboembolism among patients with AF. ${ }^{1}$ However, according to a Danish nationwide cohort study, patients who had a $\mathrm{CHA}_{2} \mathrm{DS}_{2}$ VASC score of 0 and did not have anticoagulant therapy had a stroke risk of $0.64 \%$ per year. ${ }^{12}$ Another study investigated the echocardiographic findings associated with higher risk of thromboembolism with transthoracic echocardiography (TTE) among patients with AF, and 32 out of 113 patients with $\mathrm{CHA}_{2} \mathrm{DS}_{2}$ VASC score of $0-1$ had high TEE risk. In this low $\mathrm{CHA}_{2} \mathrm{DS}_{2}$ VASC group, there was a significant SUA level difference between patients with TEE-positive and -negative risks. ${ }^{13}$ These results

Table 3

Evaluation of factors affecting stroke/TIA history with multivariate logistic regression analysis.

\begin{tabular}{lll}
\hline Variable & OR $(95 \% \mathrm{CI})$ & $P$ value \\
\hline SUA $\geq 6.35 \mathrm{mg} / \mathrm{dl}$ & $81.623(17.33-384.26)$ & $<0.001$ \\
$>65$ years & $0.707(0.163-3.073)$ & 0.644 \\
Coronary artery disease & $0.322(0.071-1.461)$ & 0.142 \\
Male sex & $0.847(0.198-3.625)$ & 0.823 \\
\hline
\end{tabular}

SUA, serum uric acid. 
suggest that newer risk parameters that can be used in patients with low $\mathrm{CHA}_{2} \mathrm{DS}_{2}$ VASC score might be useful to detect patients with true low risk.

Uric acid represents a marker of oxidative stress and inflammation, but depending on the cellular environment, it may exert antioxidant or pro-oxidant effects. ${ }^{4,14}$ Prior studies reported an association between SUA level and stroke risk, indicating that stroke patients with hyperuricemia had higher early-term mortality. ${ }^{7,15}$ Studies investigating the association between SUA level and stroke risk reported controversial results. In a study by Chao et al., patients with higher SUA levels had higher stroke risk. ${ }^{2}$ Loffredo et al. conducted a study with $163 \mathrm{AF}$ patients, of whom 45 had paroxysmal AF, and showed that there was no significant difference in terms of SUA level and ischemic stroke history. ${ }^{16}$ In our cohort, which was composed entirely of paroxysmal AF patients, we showed the independent association between SUA level and stroke/transient ischemic attack.

Most of the thromboembolism cases observed in AF patients originate from left atrial appendegeal thrombus. It is widely accepted that the presence of spontaneous echo contrast in the left atrium and decreased left atrial appendegeal velocity were associated with thrombus formation in the left atrium and thromboembolic events. ${ }^{17,18}$ In an experimental study, AF patients had 4.4 times higher XO enzymatic activity in left atrial appendix when compared to a control group. ${ }^{19}$ Cellular damage caused by increased oxidative stress causes atrial remodeling. ${ }^{20}$ Prior studies showed a correlation between the left atrial diameter, which is accepted to be the conventional marker of atrial structural remodeling, and SUA levels. ${ }^{21}$

In patients with $\mathrm{AF}$, evidence that elevation of uric acid was associated with the presence of SEC/thrombus and reduced functions of left atrial appendage are available. ${ }^{22-24}$ In study conducted by Liao et al., they reported that SUA value of $0.67 \mu \mathrm{mol} / \mathrm{L}$ and above associated with presence of SEC in the left atrium in patients with atrial fibrillation. ${ }^{22}$ Also, in a study by Tang et al., it has been reported to association with elevation of SUA and presence of thrombus in the left atrium in patients with nonvalvular atrial fibrillation. ${ }^{23}$ Additionally, in a study which followed 7601 patients with nonvalvular AF for three years, it was reported that hyperuricemia is a significant risk factor for stroke development by Chao et al. $^{2}$ Letsas et al. showed an association between high SUA levels and permanent AF, in addition to nonsignificant increase in SUA levels in paroxysmal AF group when compared to controls. This difference between AF subgroups might in part be explained by the structural nature of remodeling in permanent AF as opposed to its electrical nature in paroxysmal form. Moreover, the number of paroxysmal AF patients was low in this study, which might explain the lack of significance between groups. ${ }^{21}$

Anticoagulant treatment started after the diagnosis of AF decreases the risk of stroke by $40 \%$ as opposed to antiplatelet therapy alone. ${ }^{25}$ Therefore, recognizing AF patients with high stroke risk is important to initiate maximal stroke preventive therapy. There are several papers in the literature which reported paroxysmal AF patients developing cerebral embolism despite low $\mathrm{CHA}_{2} \mathrm{DS}_{2}$ VASC scores. Assessment of SUA levels might be useful in those patients to predict the risk. Initiating anticoagulant therapy in addition to XO inhibition with allopurinol might decrease the risk of stroke among patients with hyperuricemia.

\section{Limitations}

The possible limitations of the present study include that it was a single-center experience and included a small number of patients. Furthermore, we could not obtain Holter ECG recordings in all patients and we did not perform carotid artery Doppler investigation in all patients. The use of diuretics for management of hypertension might have affected SUA levels. Since we could not obtain data regarding number of attacks per year to determine AF load, we failed to demonstrate the relationship between stroke risk and AF load.

\section{Conclusion}

Since the mortality and morbidity of stroke associated with AF is high, recognizing paroxysmal AF patients with high stroke risk is very important. Considering that it is an easy-to-use, cheap and widely accessible measurement, SUA levels can be used to predict thromboembolic risk in patients with paroxysmal $\mathrm{AF}$ in addition to $\mathrm{CHA}_{2} \mathrm{DS}_{2}$ VASC scores.

\section{Conflict of interest}

None declared.

\section{References}

1. Camm AJ, Kirchhof P, Lip GY, et al. Guidelines for the management of atrial fibrillation: The Task Force for the Management of Atrial Fibrillation of the European Society of Cardiology (ESC). Eur Heart J 2010;31:2369-2429.

2. Chao TF, Liu CJ, Chen SJ, et al. Hyperuricemia and the risk of ischemic stroke in patients with atrial fibrillation-Could it refine clinical risk stratification in AF? Int J Cardiol 2014;170:344-349.

3. Komatsu T, Tachibana H, Satoh Y, et al. Relationship between CHA(2)DS(2)-VASc scores and ischemic stroke/cardiovascular events in Japanese patients with paroxysmal atrial fibrillation not receiving anticoagulant therapy. J Cardiol 2012;59: 321-328.

4. Glantzounis GK, Tsimoyiannis EC, Kappas AM, et al. Uric acid and oxidative stress. Curr Pharm Des 2005;11:4145-4151.

5. Bergamini C, Cicoira AJ, Rossi A, et al. Oxidative stress and hyperuricemia: Pathophysiology, clinical relevance, and therapeutic implications in chronic heart failure. Eur J Heart Fail 2009;11:444-452.

6. Korantzopoulos P, Kolettis TM, Galaris D, et al. The role of oxidative stress in the pathogenesis and perpetuation of atrial fibrillation. Int J Cardiol 2007;115: 135-143.

7. Karagiannis A, Mikhailidis DP, Tziomalos K, et al. Serum uric acid as an independent predictor of early death after acute stroke. Circ J 2007;71:1120-1127.

8. Camm AJ, Kirchhof P, Lip GY, et al. Guidelines for the management of atrial fibrillation: The Task Force for the Management of Atrial Fibrillation of the European Society of Cardiology (ESC). Eur Heart J 2010;31:2369-2429.

9. Sacco RL, Kasner SE, Broderick JP, et al. An updated definition of stroke for the 21st century: A statement for healthcare professionals from the American Heart Association/American Stroke Association. Stroke 2013;44:2064-2089.

10. Lang RM, Bierig M, Devereux RB, et al. Chamber Quantification Writing Group; American Society of Echocardiography's Guidelines and Standards Committee; European Association of Echocardiography. Recommendations for chamber quantification: A report from the American Society of Echocardiography's Guidelines and Standards Committee and the Chamber Quantification Writing Group developed in conjunction with the European Association of Echocardiography, a branch of the European Society of Cardiology. J Am Soc Echocardiogr 2005;18: 1440-1463.

11. Mattle HP. Long-term outcome after stroke due to atrial fibrillation. Cardiovasc Dis 2003;16(Suppl 1):3-8.

12. Taillandier S, Olesen JB, Clémenty N, et al. Prognosis in patients with atrial fibrillation and CHA2DS2-VASc score $=0$ in a community-based cohort study. J Cardiovasc Electrophysiol 2012;23:708-713.

13. Numa S, Hirai T, Nakagawa K, et al. Hyperuricemia and transesophageal echocardiographic thromboembolic risk in patients with atrial fibrillation at clinically lowintermediate risk. Circ J 2014;78:1600-1605.

14. Strazzullo P, Puig JG. Uric acid and oxidative stress: Relative impact on cardiovascular risk? Nutr Metab Cardiovasc Dis 2007:17:409-414.

15. Strasak AM, Kelleher CC, Brant LJ, et al, VHM\&PP Study Group. Serum uric acid is an independent predictor for all major forms of cardiovascular death in 28.613 elderly women: A prospective 21-year follow-up study. Int J Cardiol 2008;125: 232-239.

16. Loffredo L, Violi F, Fimognari FL, et al. The association between hyperhomocysteinemia and ischemic stroke in patients with non-valvular atrial fibrillation. Haematologica 2005;90:1205-1211.

17. Sasahara E, Nakagawa K, Hirai T, et al. Clinical and transesophageal echocardiographic variables for prediction of thromboembolic events in patients with nonvalvular atrial fibrillation at low-intermediate risk. J Cardiol 2012;60:484-488.

18. Iwama M, Kawasaki M, Tanaka R, et al. Left atrial appendage emptying fraction assessed by a feature-tracking echocardiographic method is a determinant of thrombus in patients with nonvalvular atrial fibrillation. J Cardiol 2012;59: 329-336.

19. Dudley Jr SC, Hoch NE, McCann LA, et al. Atrial fibrillation increases production of superoxide by the left atrium and left atrial appendage: Role of the NADPH and xantine oxidases. Circulation 2005;112:1266-1273.

20. Yang KC, Dudley SC, Jr. Oxidative stress and atrial fibrillation: Finding a missing piece to the puzzle. Circulation 2013;128:1724-1726.

21. Letsas KP, Korantzopoulos P, Filippatos GS, et al. Uric acid elevation in atrial fibrillation. Hell J Cardiol 2010;51:209-213. 
22. Liao HT, Liu FZ, Xue YM, et al. Predictive value of serum uric acid on left atrial spontaneous echo contrast in non-valvular atrial fibrillation patients. J Geriatr Cardiol 2015;12:641-646.

23. Tang RB, Dong JZ, Yan XL, et al. Serum uric acid and risk of left atrial thrombus in patients with nonvalvular atrial fibrillation. Can J Cardiol 2014;30: 1415-1421.
24. Celik M, Yalcinkaya E, Yuksel UC, et al. Increased serum uric acid levels are correlated with decreased left atrial appendage peak flow velocity in patients with atrial fibrillation. Med Princ Pract 2015;24:263-268.

25. Hart RG, Pearce LA, Aguilar MI. Meta-analysis: Anti-thrombotic therapy to prevent stroke in patients who have nonvalvular atrial fibrillation. Ann Intern Med 2007;146: 857-886. 